\title{
Measurement of Health-Related Quality of Life Among Colorectal Cancer Patients Using the Vietnamese Value Set of the EQ-5D-5L
}

This article was published in the following Dove Press journal: Patient Preference and Adherence

Binh Thang Tran, (D) ${ }^{1,2}$ Nhu Hiep Pham, ${ }^{3}$ Thanh Xuan Nguyen, ${ }^{3}$ Kui Son Choi, iD ${ }^{1,4}$ Dae Kyung Sohn, (D) ',5 Sun-Young Kim, (iD) Jae Kyung Suh, iD ${ }^{6}$ Thuy Duyen Nguyen, (D) ${ }^{1,7}$ Van Sang Phan, ${ }^{2}$ Dinh Trung Tran, (D) ${ }^{8}$ The Thanh Nguyen, (D) ${ }^{9}$ Thi Thanh Binh Nguyen, (iD) ${ }^{10}$ Minh Tu Nguyen, (D) " Jin-Kyoung Oh (D) I,12

'Department of Cancer Control and Population Health, Graduate School of Cancer Science and Policy, National Cancer Center, Goyang, Republic of Korea; ${ }^{2}$ Faculty of Public Health, Hue University of Medicine and Pharmacy, Hue University, Hue City, Vietnam; ${ }^{3}$ Department of Abdominal Emergency and Pediatric Surgery, Hue Central Hospital, Hue City, Vietnam; ${ }^{4}$ National Cancer Control Institute; National Cancer Center, Goyang, Republic of Korea; ${ }^{5}$ Center for Colorectal Cancer, Research Institute and Hospital, National Cancer Center, Goyang, South Korea; ${ }^{6}$ Division of Healthcare Technology Assessment Research, National Evidence-Based Healthcare Collaborating Agency, Seoul, Korea; ${ }^{7}$ Center for Population Health Sciences, Hanoi University of Public Health, Hanoi, Vietnam; ${ }^{8}$ Faculty of Public Health, Da Nang University of Medical Technology and Pharmacy, Da Nang City, Vietnam; ${ }^{9}$ Department of Endoscopy and Functional Exploration, Da Nang Oncology Hospital, Da Nang City, Vietnam; ${ }^{10}$ Department of Pediatrics, Hue University of Medicine and Pharmacy, Hue University, Hue City, Vietnam;

" Undergraduate Training Office, Hue University of Medicine and Pharmacy, Hue University, Hue City, Vietnam; ${ }^{12}$ Division of Cancer Prevention \& Early Detection, National Cancer Center, Goyang, Republic of Korea

Correspondence: Jin-Kyoung Oh

Department of Cancer Control and Population Health, Graduate School of Cancer Science and Policy, National Cancer Center, 323 Ilsan-Ro, Ilsandong-Gu, Goyang-si, Gyeonggi-do 4I0-

769, Republic of Korea

Tel +82-3I-920-292I

Fax +82-31-920-2929

Email jkoh@ncc.re.kr
Purpose: Health-related quality of life (HRQoL) is an important construct in clinical settings, and it is crucial that it should be properly measured. As the EuroQol-5-dimensions-5 levels (EQ-5D-5L) is more effective for such measurement than the 3-level model, data on economic models, clinical studies, and public health evaluations previously collected through the EQ-5D-3L need to be revaluated using the EQ-5D-5L. This study evaluated colorectal cancer (CRC) patients' HRQoL scores using the Vietnamese EQ-5D-5L value set. Patients and Methods: The cross-sectional study included CRC patients treated at a tertiary public hospital. HRQoL was assessed using the EQ-5D-5L, and HRQoL utility scores were calculated using the Vietnamese value set. Tobit regression examined factors associated with HRQoL.

Results: The analysis included $197 \mathrm{CRC}$ patients. Ages ranged from 20 to 87 years $(\mathrm{M}=$ $57.64, \mathrm{SD}=13.5) ; 42.2 \%$ and $57.8 \%$ were diagnosed with cancer of the colon or rectum/ anus, respectively. Mean EQ-5D-5L was 0.561 (range, -0.5115 to 1). Most participants experienced anxiety/depression (88\%), followed by pain/discomfort $(87 \%)$, mobility $(71 \%)$, usual activity (69\%), and self-care (67\%). Advanced CRC stage (stage II: $\beta-0.303$, se 0.08 ; stage III: $\beta-0.305$, se 0.07 ; stage IV: $\beta-0.456$, se 0.07$)$ and surgery $(\beta-0.113$, se 0.05$)$ were negatively associated with EQ-5D-5L scores. Advanced education (high school: $\beta 0.273$, se 0.07); college/vocational: $\beta 0.134$ se 0.05 ; university/higher: Coef 0.213 , se 0.08 ;) and older age (age group $35-44$ : $\beta 0.253$, se $0.10 ; 45-54$ : $\beta 0.327$, se 0.09 ; $55-64$ : $\beta 0.355$ se 0.09 ; $65+$ $\beta 0.204$, se 0.09 ) were positively associated with EQ-5D-5L scores.

Conclusion: Patients in advanced CRC stages or undergoing surgery experienced lower HRQoL and higher prevalence of anxiety/depression and pain/discomfort. Older age and high educational attainment predicted high HRQoL. This study provides information on CRC patients' health utility based on various patient characteristics, which can be used in future economic evaluations.

Keywords: HRQoL, EQ-5D-5L, utility, colorectal cancer, Vietnam

\section{Introduction}

Globally, colorectal cancer (CRC) is the third most diagnosed type of cancer and the fifth most common cause of cancer-related death. Although highly developed countries have shown stable or decreasing prevalence trends in combination with higher survival rates, $\mathrm{CRC}$ incidence and mortality continue to be a burden in many developing countries, such as Vietnam. In 2017, the CRC incidence in Vietnam was 21 persons/ 100,000 , with a death rate of 13.9 persons/100,000. ${ }^{1-3}$ These differences are partially owing to the different levels of societal and economic development between countries, 
as well as differences regarding population aging, adoption of a westernized diet, lifestyle, and cancer control policy priorities. $^{2-5}$ Moreover, although CRC incidence and mortality rates continue to rise in low-middle income countries, proper diagnosis and treatment for this type of cancer is lacking in these nations. ${ }^{6}$

Further, more than $80-90 \%$ of patients are diagnosed with CRC after it is already at an advanced stage, resulting in significant health and economic burdens for patients and their families. ${ }^{7}$ Additionally, general cultural beliefs (eg, seeking help from traditional healers or fortune-tellers) and misconceptions about cancer outcomes in late diagnosis and treatment, ${ }^{8}$ when coupled with the effects of complex treatment regimens (sometimes requiring the combination of surgery, radiotherapy, and systemic chemotherapy), lead to reduced quality of life for patients and their families. Thus, a comprehensive understanding of CRC patients' health-related quality of life (HRQOL) is crucial for health professionals and decision-makers to be able to effectively control this type of cancer.

Health utility refers to a person's preferred outcomes regarding their own health; to measure this construct, researchers have mostly analyzed individual preferences through a wide array of methods - visual analog scales, time trade-offs, or standard gamble techniques. ${ }^{9,10}$ By measuring HRQOL, we believe that we may be able to not only analyze whether patients' desired treatment outcomes reflect their survival rates but also their ability to live comfortable, disease-free lives. Hence, given the rising concerns regarding CRC, an index based on patients' health-related quality of life could potentially improve patient experience and care. Furthermore, research using health technology assessments and in the health economics field have increasingly utilized patient-reported outcome measures, which provide essential information to plan interventions that can help guide patients' improvement efforts and enhance system-level policies. $^{9,10}$

There are diverse instruments to measure HRQOL, each with its own strengths and limitations. Among them, numerical algorithms have been developed to map disease-specific questionnaires to the EuroQol Group Five-Dimensional (EQ-5D), a standardized instrument for measuring generic health status, values in cancer groups. ${ }^{11-14}$ The EQ-5D is one of the most commonly used instruments to describe and assess HRQoL based on public preference; it has two versions: the 3 level (3L) and 5 level (5L). ${ }^{15}$ A study using a sizeable multinational dataset showed that, compared with the $3 \mathrm{~L}$, the $5 \mathrm{~L}$ is more accurate and preferred regarding both study results and participant responses. ${ }^{14,16}$ Considering this, the National Institute for Health and Care Excellence (NICE) in the United Kingdom has suggested using the $5 \mathrm{~L}$, instead of the 3L, in economic models, clinical studies, and public health evaluations. ${ }^{17}$ Furthermore, the EQ-5D-5L is particularly useful for cancer research, because patients are usually experiencing severe health psychological or physical conditions, and are thus unable to answer lengthy questionnaires. $^{18}$

In Vietnam, the first study to use the EQ-5D-5L was conducted among the general adult population to validate the instrument; however, it used the value set from the Thai EQ-5D-5L. ${ }^{19}$ Moreover, the Vietnamese EQ-5D-5L was recently developed and validated using the EuroQoL Group's standardized protocol on a nationally representative sample of the Vietnamese population, and its first instruments provided promising local information on health economics in Vietnam. ${ }^{20}$ Nonetheless, to the best of the researchers' knowledge, there is no study to date on all cancer patients' HRQoL in Vietnam. Therefore, this study aimed to assess CRC patients' HRQoL and determine the associated factors using the EQ-5D-5L in a public tertiary hospital in the central region of Vietnam.

\section{Patients and Methods Participants}

This was a cross-sectional study conducted with CRC patients aged 20 years and older at Hue Central Hospital $(\mathrm{HCH}) . \mathrm{HCH}$ is a tertiary referral hospital and the thirdlargest public hospital, located in the North Central Coast region of Vietnam, in the approximate center of the country. Although the region has 1.2 million residents, $\mathrm{HCH}$ provides healthcare for more than 15 million people in Central Vietnam. ${ }^{21}$ The hospital's oncology center provides specialist care to cancer patients in the corresponding and neighboring provinces. ${ }^{21}$

The inclusion criteria were patients who had complete clinical data, were diagnosed with primary CRC within the last four years, and were able to answer the questionnaire. Exclusion criteria included patients who were unable to complete the questionnaire and patients who regularly experienced fatigue or psychological difficulty.

\section{Sample Size Determination and Participant Recruitment}

With the aim of estimating the overall mean score of the EQ 5D-5L health utility for CRC patients, we assumed the 
expected sample standard deviation (SD) would be 0.2456 , recalculated based on the EQ-5D-5L value set. ${ }^{20} \mathrm{~A}$ desired precision of $3 \%$ was set, which indicated precisely how to estimate the mean of the population and the $95 \%$ level of confidence for t-distribution. ${ }^{22}$ An initial sample size of 261 patients was required; however, according to an annual report, the total number of CRC inpatients at $\mathrm{HCH}$ was about 700 ; therefore, we needed to adjust to a finite population in this case. Therefore, a sample size of 190 would suffice. In addition, we assumed the refusal rate to be about $5 \%$. Thus, the final sample size was 200 patients.

Accordingly, 200 eligible CRC inpatients were identified at HCH between July and December 2019 using convenience sampling, and then invited to participate in the study. Face-to-face interviews with patients were conducted by a medical school student and a well-trained nurse in the same hospital. Prior to the interviews, participants were informed about this study via flyers; they also received detailed information from the interviewers, after which they provided signed informed consent. Information about participants' socio-demographic characteristics was recorded on the EQ-5D-5L questionnaire. Information on clinical variables was further retrieved from the hospital's medical records or by consultation with their respective physicians; this information included the CRC stage and treatment regimen. Interviews lasted approximately 12-15 minutes per patient.

\section{Measurements}

\section{Independent Variables}

Questions on the patients' socio-demographic characteristics included: age, gender (male, female), marital status (single/widow; married), education (primary school or lower, secondary school, high school, college/vocational, and university/higher), occupation (agriculture, business/ employment, retired/elderly, housework, and freelancers/ unemployed), and economic status (monthly income by government classification: poor [VND $\leq 900,000, \$$ US 45]; near-poor [VND 900,000-1,300,000, \$ US 45 to 65]; and fair: [ $\geq$ VND 1,500,000; \$ US 75]).

Regarding CRC stage, participants were classified in accordance with the Union for International Cancer Control (UICC) TNM classification ${ }^{23}$ stages I, II, III, and $\mathrm{IV}$; their staging system also has an "undifferentiated stage," which we classified as "unknown." In this study, CRC was divided into colon and rectum cancer, and the definition was based on patients' medical records, which included this classification. The treatment regimen was based on patients' current treatment (at the time of the study), including chemotherapy, radiotherapy, surgery, and other types of therapy (internal medicine).

\section{Dependent Variables}

The EQ-5D-5L has been recently validated within the Vietnamese general population; ${ }^{20}$ thus, our study evaluated patients' HRQoL using this instrument. This scale has five dimensions: mobility, self-care, usual activities, pain/discomfort, and anxiety/depression. ${ }^{15}$ Each dimension is rated on a 5 -point Likert scale $(1=$ no problem; $2=$ slight problem; $3=$ moderate problem; $4=$ severe problem; $5=$ extreme problem). Originally, the combination of the reported levels for the five dimensions of this scale generated 3125 possible health states, with the healthiest state being "11,111" (showing "no problem at all") and most severe state being "55,555" (showing "extreme problems"). In our study, each health state was scored based on the Vietnamese EQ-5D-5L value set, which was constructed in a standardized study by Mai et al, to estimate each participant's health utility score. These scores ranged from -0.5115 to $1 .^{20}$

\section{Statistical Analysis}

After excluding three patients who declined to participate, data from 197 patients were included in the final analysis. Normal distribution for the EQ-5D-5L utility score was computed using the Shapiro-Wilk test $(\mathrm{p}<0.05)$, and the results indicated that it followed a non-normal distribution. Results of statistical analyses regarding the health utility scores were presented as means, SD, and medians. Regarding the univariate relationship between health utility scores and independent variables, non-parameter tests (Kruskal-Wallis and Wilcoxon's rank-sum test) were utilized.

Regarding censored data for outcome variables, as health utility scores ranged from -0.5115 to 1 in this study, the Tobit regression model was an eligible statistical test for examining the associations between the EQ-5D-5L utility score and other independent variables. ${ }^{24}$ All variables in the univariate analysis were included in the multivariate model. All data analysis was performed using the $\mathrm{R}$ language. A p-value less than 0.05 was considered statistically significant.

\section{Ethical Approval}

The study was conducted in accordance with the Declaration of Helsinki and approved by the review 
board of Da Nang University of Medical Technology and Pharmacy, Vietnam (Code: 0259/QĐ-HĐĐNCYSH), and it was performed with the agreement of the Board of Directors of the Hue Central Hospital, Hue city, Vietnam. All participants provided written informed consent.

\section{Results}

The socio-demographic characteristics of the 197 participants (98.5\% participant rate) are shown in Table 1. Regarding their socio-demographics, participants' mean age was 57.7 (SD 13.5), with $54.8 \%$ identifying as male. Most participants were married $(86.8 \%)$, about one-third had completed high school (34.6\%), 40\% were farmers, and most had an economic status of poor $(55.8 \%)$ or fair $(42.6 \%)$.

Regarding clinical characteristics, most participants had been diagnosed with CRC at stage III (48.2\%) or stage IV (24.9\%); 57\% were diagnosed with rectum cancer. The average time that had passed from participants' diagnosis to their participation in this survey was 6.4 months. Most respondents had been diagnosed within two years $(78.2 \%$ in $2019 ; 15.2 \%$ in 2018$)$ of their participation in the interview, and most were receiving treatment with chemotherapy and surgery (76.1\%). The distribution of the EQ-5D-5L utility scores is shown in Figure 1; the shape of the curve was skewed to the right, at a higher value.

Table 1 also compares the summary statistics for participants' EQ-5D-5L utility scores based on their sociodemographic and clinical characteristics. The mean and median of CRC patients' EQ-5D-5L utility scores were 0.561 (SD, 0.27) and 0.582 (Interquartile range, IQR, -0.21 to 0.78 ), respectively. There were statistically significant differences in patients' utility scores based on the following characteristics: age group $(p=0.01)$, education $(\mathrm{p}=0.01)$, cancer type $(\mathrm{p}=0.03)$, and CRC stage $(\mathrm{p}=$ $0.0001)$. No significance was found among the other variables with utility scores $(\mathrm{p}>0.05)$.

The proportion of each dimension of the EQ-5D-5L by CRC stage is shown in Figure 2. Most participants experienced anxiety/depression (88\%), which went from slight to extreme levels; this was followed by pain/discomfort (87\%), mobility (71\%), usual activity (69\%), and selfcare $(67 \%)$. There were similar patterns in anxiety/depression among participants at CRC stages I and II (76.5\% and $91.2 \%$, respectively), while pain/discomfort was the most common among participants at CRC stages III and IV (91.6\% and $91.8 \%$, respectively).
The factors associated with participants' EQ-5D-5L utility scores extracted through the Tobit regression model are shown in Table 2 . The model confirmed that older age $(35-44$ years, $\beta=0.25, \mathrm{p}=0.01 ; 45-54$ years, $\beta$ $=0.327, \mathrm{p}=0.001 ; 55-64$ years, $\beta=0.355, \mathrm{p}=0.001$; and $65+$ years, $\beta=0.204, p=0.029)$ and higher levels of education (high school, $\beta=0.273, \mathrm{p}=0.0001$; college/ vocational, $\beta=0.134, \mathrm{p}=0.008$; university/higher, $\beta=$ $0.213, \mathrm{p}=0.005)$ were significantly associated with higher EQ-5D-5L utility scores, whereas having late-stage CRC (stage II, $\beta=-0.303, p=0.0001 ;$ III, $\beta=-0.305, p=$ $0.0001 ; \mathrm{IV}, \beta=-0.456, \mathrm{p}=0.0001)$ and receiving surgical treatments $(\beta=-0.113, \mathrm{p}=0.019)$ were significantly associated with lower EQ-5D-5L utility scores. Other variables, namely, gender, jobs, economic status, type of cancer, and year since diagnosis, did not show any significance association with EQ-5D-5L utility scores $(\mathrm{p}>0.05)$.

\section{Discussion}

To the researchers' knowledge, this was the first study in Vietnam to assess CRC patients' EQ-5D-5L utility scores. The results provided essential evidence regarding CRC patients' health-related quality of life and health utility, which may be applied by policymakers and other relevant stakeholders when making health-related economic evaluations. Subsequently, these evaluations can serve as background information for policy-related decisionmaking and/or clinical management, as they can provide evidence regarding specific indicators of CRC patients' health utility by age group and cancer stage. Moreover, this study can also equip stakeholders with information on a wide array of socio-demographic and clinical factors associated (and not associated) with CRC patients' health utility. In 2020, the Vietnamese Ministry of Health actively worked on the guidelines on health technology assessment, and the EQ-5D-5L was advised to use in health economic evaluation. However, it was still underdeveloped before publishing the official documents. ${ }^{25}$ Nonetheless, it has been recommended in other countries (such as the UK). Since 2008 the National Institute for Health and Care Excellence (NICE) has advised the use of a shorter, generic QoL questionnaire - the EuroQoL EQ-5D-5L - to enable standardisation of QoL measures across varied conditions and populations. $^{26}$

The overall mean utility score for CRC patients in our study was 0.561 , which was lower than that of the general adult population in Vietnam, 0.91. ${ }^{19}$ Previous studies using 
Table I General Characteristics of CRC Patients $(n=197)$ and Summary Statistics for the EQ-5D-5L Utility Score by Participant and Clinical Characteristics

\begin{tabular}{|c|c|c|c|c|c|}
\hline Variables & Frequency & Mean & SD & Median & p-value \\
\hline All & 197 & $\begin{array}{l}0.56 I(\min , \max : \\
-0.5 \mid I 5, I)\end{array}$ & 0.27 & $\begin{array}{l}0.582 \\
(\mathrm{IQR},-0.209 \text { to } 0.782)\end{array}$ & NA \\
\hline \multicolumn{6}{|l|}{ Gender } \\
\hline Men & 108 & 0.559 & 0.29 & 0.548 & 0.88 \\
\hline Women & 89 & 0.564 & 0.25 & 0.599 & \\
\hline \multicolumn{6}{|l|}{ Marital status } \\
\hline Single/widow & 26 & 0.492 & 0.31 & 0.505 & 0.22 \\
\hline Married & $|7|$ & 0.572 & 0.26 & 0.593 & \\
\hline \multicolumn{6}{|l|}{ Age group } \\
\hline $20-34$ & 10 & 0.447 & 0.37 & 0.475 & 0.01 \\
\hline $35-44$ & 23 & 0.602 & 0.25 & 0.611 & \\
\hline $45-54$ & 39 & 0.596 & 0.18 & 0.593 & \\
\hline $55-64$ & 66 & 0.639 & 0.20 & 0.667 & \\
\hline $65+$ & 59 & 0.454 & 0.34 & 0.474 & \\
\hline \multicolumn{6}{|l|}{ Education } \\
\hline Primary school and less (grade 5 or less) & 58 & 0.472 & 0.30 & 0.542 & 0.01 \\
\hline Secondary school (Grade 6-9) & 71 & 0.549 & 0.26 & 0.558 & \\
\hline High school (grade 10 or 12) & 13 & 0.692 & 0.22 & 0.800 & \\
\hline College/vocational & 39 & 0.635 & 0.20 & 0.652 & \\
\hline University/higher & 16 & 0.651 & 0.30 & 0.782 & \\
\hline \multicolumn{6}{|l|}{ Jobs } \\
\hline Agricultures & 77 & 0.591 & 0.25 & 0.604 & 0.24 \\
\hline Business/Employment & 36 & 0.608 & 0.20 & 0.608 & \\
\hline Retired/Elderly/Housework & 59 & 0.492 & 0.32 & 0.542 & \\
\hline Freelancers/Unemployed & 25 & 0.565 & 0.27 & 0.467 & \\
\hline \multicolumn{6}{|l|}{ Economic status (Average monthly income) } \\
\hline Poor (VND $\leq 900,000)$ & 3 & 0.633 & 0.20 & 0.667 & 0.92 \\
\hline Near-poor (VND 900,000-1,300,000) & 110 & 0.555 & 0.29 & 0.548 & \\
\hline Fair $(\geq \mathrm{VNDI}, 500,000)$ & 84 & 0.566 & 0.25 & 0.599 & \\
\hline \multicolumn{6}{|l|}{ Cancer } \\
\hline Colon $(\mathrm{CI})$ & 85 & 0.600 & 0.27 & $0.64 I$ & 0.03 \\
\hline Rectum (C20) & 112 & 0.532 & 0.27 & 0.543 & \\
\hline \multicolumn{6}{|l|}{ Year of diagnosis } \\
\hline 2019 & 154 & 0.551 & 0.28 & 0.593 & 0.77 \\
\hline 2018 & 30 & 0.584 & 0.23 & 0.540 & \\
\hline Before 2018 & 13 & 0.628 & 0.21 & 0.720 & \\
\hline \multicolumn{6}{|l|}{ CRC Stage } \\
\hline I & 17 & 0.806 & 0.12 & 0.821 & 0.0001 \\
\hline II & 34 & 0.565 & 0.35 & 0.667 & \\
\hline III & 95 & 0.571 & 0.21 & 0.548 & \\
\hline IV & 49 & 0.458 & 0.30 & 0.485 & \\
\hline Undifferentiated & 2 & 0.480 & 0.09 & 0.480 & \\
\hline \multicolumn{6}{|l|}{ Treatment status } \\
\hline Chemotherapy & 103 & 0.578 & 0.25 & 0.604 & 0.53 \\
\hline Radiotherapy & 31 & 0.479 & 0.34 & 0.485 & \\
\hline
\end{tabular}

(Continued) 
Table I (Continued).

\begin{tabular}{|l|l|l|l|l|}
\hline Variables & Frequency & Mean & P-value \\
\hline Surgery & 47 & 0.564 & Median & 0.28 \\
Other treatment's regimes (Internal medicine/no & 16 & 0.604 & 0.24 \\
direction of treatment & & 0.577 & & \\
\hline
\end{tabular}

Notes: Bold: statistical significance. Household economic status defined as per the Vietnamese Government's (No. 59/20I5/QDD-TTg) categorization based on average

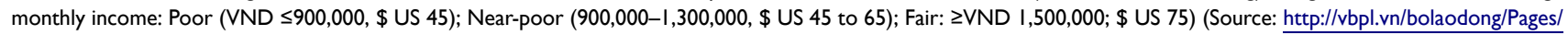
vbpq-toanvan.aspx?!temID=92948).

Abbreviations: NA, not available; IQR, interquartile range.

the EQ-5D-5L in CRC in other countries reported the scores higher than that of the current study: 0.81 in South Korea in $2012,{ }^{11} 0.710$ in Iran in $2017,{ }^{13} 0.87$ in Japan in $2017,{ }^{27}$ and 0.617 in China (the most recent study) in $2018 .{ }^{28}$ This difference may be related to the sample percentages for each cancer stage; in our study, most were at the later stages (73\% patients in stages III and IV) of cancer development, which may explain the overall low utility score. This explanation is reasonable, as the Chinese study had a sample in which half the patients were at later CRC stages, similar to our study. Participants' mean utility scores by stage were $0.77,0.66,0.56$, and 0.5 for Stage I to IV, respectively, ${ }^{28}$ with the latter two being similar to the overall mean utility score in our study. Further, this difference may also be partially explained by the use of the Vietnamese value set of the EQ-5D-5L, and between-study gaps regarding clinical context, healthcare systems, treatment technological advances, and CRC patients' socio-economic status.

Nevertheless, higher HRQoL scores in patients with a longer time interval since diagnosis were also found in previous study on CRC patients in Hong Kong. ${ }^{29}$ However, this finding was not statistically significant in our study, and the time interval time from diagnosis to interview differed between our study and previous studies conducted in Hong Kong (47 months), ${ }^{28}$ China (26 days), ${ }^{30}$ Finland (6-8 months), ${ }^{31}$ and England (at least 12 months). ${ }^{32}$ In addition, the evidence for the linear relationship between time interval from diagnosis and outcome was inconsistent between previous studies and the current study, which could partially be explained by the differences in the measurement of interval time from diagnosis. The interval between a confirmed CRC diagnosis and the start of treatment may

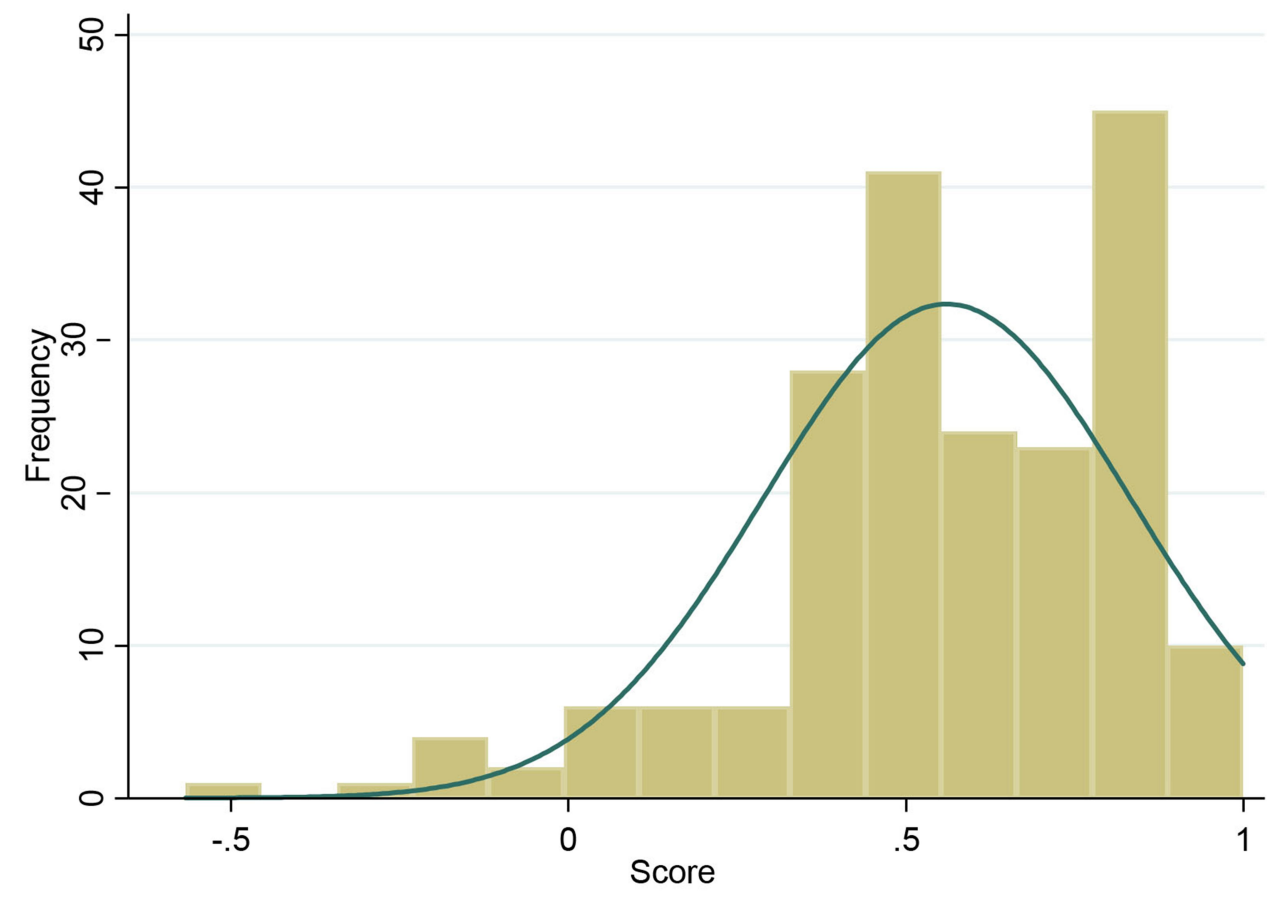

Figure I Distribution of the EQ-5D-5L utility score. 


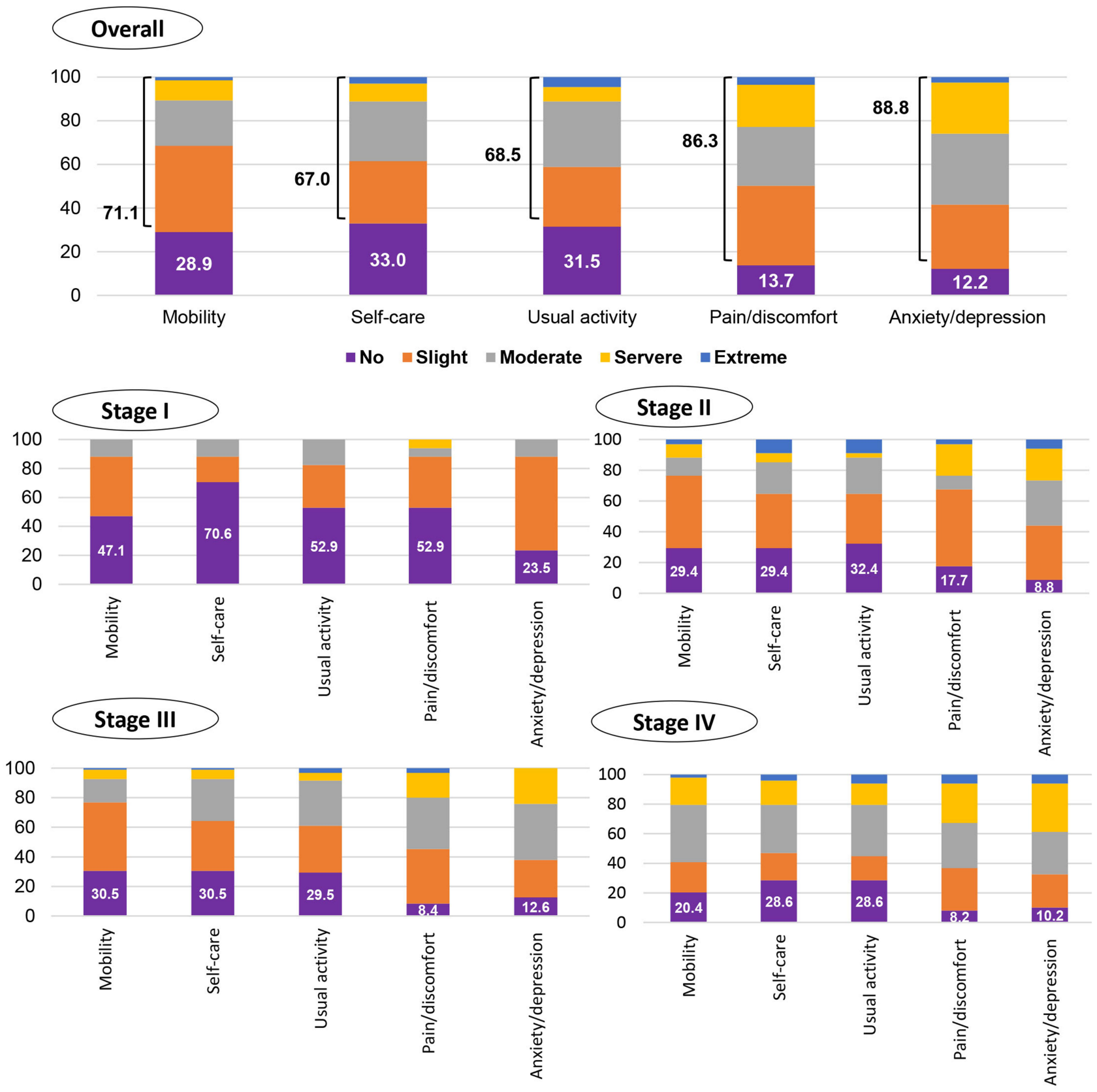

Figure 2 The proportion of levels of problem on each EQ-5D-5L dimension in the overall sample and by CRC stage (percent, \%).

influence patients' health status in different ways. ${ }^{31,33}$ A systematic review found a significant association between shorter time since diagnosis and favorable outcomes, including HRQoL, among cancer patients. ${ }^{34}$ Future studies should better define time intervals as either the duration from diagnosis or the duration from treatment to survey.

Previous studies showed a high prevalence of anxiety/ depression among CRC patients, followed by pain/ discomfort. ${ }^{11,32,35}$ These dimensions were also dominant in our study, which found that nine out of ten patients experienced anxiety/depression and, among CRC patients at later stages, pain/discomfort had the most negative influence and was more widespread. Generally, one out of four patients reported experiencing anxiety/depression and pain/discomfort at severe to extreme levels, and there were more severe-level cases in the later stages. These numbers may reflect that most participants in our study were at later stages of CRC (more than $73 \%$ were at stages III and IV), as late-stage cancer tends to worsen health status. These results corroborate the findings of Kim et al 
Table 2 Tobit Regression Model: Factors Associated with the EQ-5D-5L Utility Score

\begin{tabular}{|c|c|c|c|c|}
\hline Variables & Frequency & Coefficient & se & p-value \\
\hline \multicolumn{5}{|l|}{ Gender } \\
\hline Men & 108 & Reference & & \\
\hline Women & 89 & 0.025 & 0.03 & 0.46 \\
\hline \multicolumn{5}{|l|}{ Marital status } \\
\hline Single/widow & 26 & Reference & & \\
\hline Married & $17 \mid$ & -0.053 & 0.06 & 0.342 \\
\hline \multicolumn{5}{|l|}{ Age group } \\
\hline $20-34$ & 10 & Reference & & \\
\hline $35-44$ & 23 & 0.253 & 0.10 & 0.01 \\
\hline $45-54$ & 39 & 0.327 & 0.09 & 0.001 \\
\hline $55-64$ & 66 & 0.355 & 0.09 & 0.001 \\
\hline $65+$ & 59 & 0.204 & 0.09 & 0.029 \\
\hline \multicolumn{5}{|l|}{ Education } \\
\hline Primary school and less (grade 5 or less) & 58 & Reference & & \\
\hline Secondary school (Grade 6-9) & 71 & 0.077 & 0.04 & 0.066 \\
\hline High school (grade 10 or 12 ) & 13 & 0.273 & 0.07 & 0.0001 \\
\hline College/vocational & 39 & 0.134 & 0.05 & 0.008 \\
\hline University/higher & 16 & 0.213 & 0.08 & 0.005 \\
\hline \multicolumn{5}{|l|}{ Jobs } \\
\hline Agriculture & 77 & Reference & & \\
\hline Business/Employment & 36 & -0.019 & 0.06 & 0.738 \\
\hline Retired/Elderly/Housework & 59 & -0.061 & 0.04 & 0.134 \\
\hline Freelancers/Unemployed & 25 & -0.067 & 0.06 & 0.228 \\
\hline \multicolumn{5}{|l|}{ Economic status (Average monthly income) } \\
\hline Poor (VND $\leq 900,000)$ & 3 & Reference & & \\
\hline Near-poor (VND 900,000-1,300,000) & 110 & -0.074 & 0.14 & 0.602 \\
\hline Fair $(\geq \mathrm{VNDI}, 500,000)$ & 84 & -0.038 & 0.14 & 0.791 \\
\hline \multicolumn{5}{|l|}{ Cancer } \\
\hline Colon (CI8) & 85 & Reference & & \\
\hline Rectum (C20) & 112 & -0.047 & 0.04 & 0.184 \\
\hline \multicolumn{5}{|l|}{ Year of diagnosis } \\
\hline 2019 & 154 & Reference & & \\
\hline 2018 & 30 & 0.003 & 0.05 & 0.946 \\
\hline Before 2018 & 13 & 0.065 & 0.07 & 0.332 \\
\hline \multicolumn{5}{|l|}{ CRC Stage } \\
\hline 1 & 17 & Reference & & \\
\hline II & 34 & -0.303 & 0.08 & 0.00001 \\
\hline III & 95 & -0.305 & 0.07 & 0.00001 \\
\hline IV & 49 & -0.456 & 0.07 & 0.00001 \\
\hline Undifferentiated & 2 & -0.369 & 0.17 & 0.0360 \\
\hline \multicolumn{5}{|l|}{ Treatment status } \\
\hline Chemotherapy & 103 & Reference & & \\
\hline Radiotherapy & 31 & -0.054 & 0.05 & 0.285 \\
\hline Surgery & 47 & -0.113 & 0.05 & 0.019 \\
\hline Other treatment's regimes (Internal medicine/no direction of treatment & 16 & 0.039 & 0.07 & 0.548 \\
\hline
\end{tabular}

Notes: Bold: statistical significance. Household economic status defined as per the Vietnamese Government's (No. 59/2015/QDD-TTg) categorization based on average

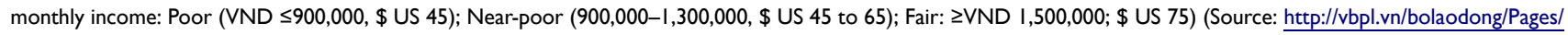
vbpq-toanvan.aspx?!temID=92948). 
(South Korea) $^{35}$ and Weidong et al (China) $)^{28}$ on HRQoL, and findings from other developing countries on pain burden in cancer treatment. ${ }^{36}$ Among rectal cancer patients in the German population, pain was a common symptom and was strongly associated with reduced QoL scores. ${ }^{37}$ For rectal cancer patients, stomas are constructed after surgical treatment and are linked to lower quality of life. ${ }^{30,32,38}$ In clinical settings, healthcare providers should endeavor to manage these problems to mitigate patients' symptoms, pain/discomfort, and anxiety/depression by implementing tailored interventions to meet patients' needs during treatment and when providing healthcare services such as palliative care, particularly for patients at advanced cancer stages. ${ }^{36}$

Overall, our results suggested that later CRC stages were negatively associated with lower health utility scores, as they showed that patients with CRC stage IV had the lowest overall scores $(0.458)$, while those with CRC stage I had the highest scores (0.806). These results were in line with previous research performed within Asian populations ${ }^{28,30}$ and populations from other regions. ${ }^{18,39}$ Furthermore, surgical procedures are usually related to patient-reported lower scores because of its association with pain, health complications, and disease severity. ${ }^{18,28,40,41}$ In this study, $23.9 \%$ of patients underwent surgery, and approximately $90 \%$ experienced or appeared to have more severe symptoms at advanced stages, which together explains the decrease in overall scores.

In our study, higher educational levels were associated with higher HRQoL scores in CRC patients, which corroborated the findings of a previous study which analyzed 1294 CRC survivors within the German population. ${ }^{42}$ This result indicated that people with higher levels of education were more conscious of their health, which, in turn, led to less exposure to environmental risk factors, frequent health checkups, and a reduced likelihood of advancement to later cancer stages. $^{43,44}$

Furthermore, being younger was recognized as a predictor of poorer HRQoL scores, a finding that was in line with recent studies on other types of cancers; specifically, one study on CRC survivors' pain in the $\mathrm{UK}^{45}$ and another in Australia. ${ }^{46}$ In general, we believe this correlation may reflect patients' life-stage expectations and demands. Moreover, a CRC diagnosis at a younger age tends to present more aggressive clinical progress, due to the early disease onset of the disease and treatment response. $^{47}$

\section{Limitations and Strengths}

This study had several limitations. First, our study used a cross-sectional design, which does not allow for the examination of causality and long-term effects of CRC on health-related quality of life. Cohort studies should be conducted in the future to examine real-world patient experiences over the course of treatment and associated factors. Second, we utilized convenience sampling. Third, as we did not account for comorbid diseases; this adds another limitation to our study; however, our inclusion criteria comprise those patients primarily diagnosed with CRC. This reflects that main symptoms are most likely due to CRC, rather than other causes. Four, since our sample was selected at a tertiary hospital, it may have resulted in a sample with patients that had more severe cases of CRC. Therefore, our results must be interpreted with caution, but can be generalized for Vietnamese CRC patients.

This study also had several strengths. First, this was the first study using the Vietnamese EQ-5D-5L value set. Second, our results can provide valuable parameters and indicators for further studies on cost-utility or costeffectiveness analysis. Third, our results add to the current evidence on CRC patients' health utility scores in Vietnam.

\section{Conclusions}

In the present study, CRC patients had poor HRQoL compared to the general population. Patients undergoing surgery for CRC and patients at advanced cancer stages experienced lower HRQoL. Most patients experienced anxiety/depression and pain/discomfort, especially in the later stages of the disease. Older age and higher levels of education were predictors of high HRQoL. This study's findings provide information on CRC patients' health utility based on various socio-demographic and clinical characteristics, and this information can be used in future economic evaluations.

\section{Acknowledgments}

We would like to thank Dr Pham Huu Tri, Dr Nguyen Minh Hanh from Hue Central Hospital, Mr Anh, Ms Hong, the whole staff in the Oncology center, and all patients, for their support during data collection.

\section{Funding}

TDN received funding from the "International Cooperation \& Education Program (\#NCCRI·NCCI 
52210-52211, 2019-2020)" of the National Cancer Center. The research project was partially supported by the National Cancer Center (grant no. 2010303).

\section{Disclosure}

The authors declare that they have no conflicts of interest.

\section{References}

1. GBD 2017 Colorectal Cancer Collaborators. The global, regional, and national burden of colorectal cancer and its attributable risk factors in 195 countries and territories, 1990-2017: a systematic analysis for the global burden of disease study 2017. Lancet Gastroenterol Hepatol. 2019;4(12):913-933. doi:10.1016/S24681253(19)30345-0

2. Nguyen SM, Deppen S, Nguyen GH, Pham DX, Bui TD, Tran TV. Projecting cancer incidence for 2025 in the 2 largest populated cities in Vietnam. Cancer Control. 2019;26(1):1073274819865274. doi:10.1177/1073274819865274

3. Fidler MM, Bray F, Vaccarella S, Soerjomataram I. Assessing global transitions in human development and colorectal cancer incidence. Int $j$ Cancer. 2017;140(12):2709-2715. doi:10.1002/ijc.30686

4. Nguyen TT, Hoang MV. Non-communicable diseases, food and nutrition in Vietnam from 1975 to 2015: the burden and national response. Asia Pac J Clin Nutr. 2018;27(1):19-28.

5. Vietnam Ministry of Health. Joint Annual Health Review 2016 Towards Healthy Aging in Vietnam. Hanoi: Vietnam Ministry of Health; 2018.

6. Arnold M, Sierra MS, Laversanne M, Soerjomataram I, Jemal A, Bray FJG. Global patterns and trends in colorectal cancer incidence and mortality. Gut.2017;66(4):683-691.

7. Van Thuan T, Tuan Anh P, Van Tu D, Thi Thanh Huong T. Cancer control in Vietnam: where are we. Cancer Control. 2016.

8. Hall A, Nguyen SM, Mackenzie L, et al. What caused my cancer? Cancer patients' perceptions on what may have contributed to the development of their cancer: a cross-sectional, cross-country comparison study. Cancer Control. 2019;26(1):1073274819863786. doi:10. $1177 / 1073274819863786$

9. Jeong K, Cairns J. Systematic review of health state utility values for economic evaluation of colorectal cancer. Health economics review. 2016;6(1):36.

10. Drummond MF, Sculpher MJ, Claxton K, Stoddart GL, Torrance GW. Methods for the Economic Evaluation of Health Care Programmes. Oxford university press; 2015.

11. Kim SH, Kim HJ, Lee SI, Jo MW. Comparing the psychometric properties of the EQ-5D-3L and EQ-5D-5L in cancer patients in Korea. Qual Life Res. 2012;21(6):1065-1073. doi:10.1007/s11136011-0018-1

12. Ameri H, Yousefi M, Yaseri M, Nahvijou A, Arab M, Akbari Sari A. Mapping EORTC-QLQ-C30 and QLQ-CR29 onto EQ-5D-5L in colorectal cancer patients. J Gastrointest Cancer. 2019.

13. Ameri H, Yousefi M, Yaseri M, Nahvijou A, Arab M, Akbari Sari A. Mapping the cancer-specific QLQ-C30 onto the generic EQ-5D-5L and SF-6D in colorectal cancer patients. Expert Rev Pharmacoecon Outcomes Res. 2019;19(1):89-96. doi:10.1080/14737167.2018.1517 046

14. Buchholz I, Janssen MF, Kohlmann T, Feng Y-S. A systematic review of studies comparing the measurement properties of the three-level and five-level versions of the EQ-5D. PharmacoEconomics. 2018;36(6):645-661. doi:10.1007/s40273-01 8-0642-5

15. EuroQol research foundation; 2019. Available from: https://euroqol. org/eq-5d-instruments/. Accessed Feb 4, 2020.
16. Janssen MF, Bonsel GJ, Is LN. EQ-5D-5L better than EQ-5D-3L? A head-to-head comparison of descriptive systems and value sets from seven countries. PharmacoEconomics. 2018;36(6):675-697. doi:10. 1007/s40273-018-0623-8

17. Pennington B, Hernandez-Alava M, Pudney S, Wailoo A. The impact of moving from EQ-5D-3L to $-5 \mathrm{~L}$ in NICE technology appraisals. Pharmaco Economics. 2019;37(1):75-84. doi:10.1007/s40273-0180701-y

18. Schwenkglenks M, Matter-Walstra K. Is the EQ-5D suitable for use in oncology? An overview of the literature and recent developments. Expert Rev Pharmacoecon Outcomes Res. 2016;16(2):207-219. doi:10.1586/14737167.2016.1146594

19. Nguyen LH, Tran BX, Hoang LQN, Tran TT, Latkin CA. Quality of life profile of general Vietnamese population using EQ-5D-5L. Health Qual Life Outcomes. 2017;15(1):199. doi:10.1186/s12955017-0771-0

20. Mai VQ, Sun S, Minh HV, et al. An EQ-5D-5L value set for Vietnam. J Quality Life Research. 2020;1-11.

21. Hue Central Hospital. Hue central hospital; 2019. Available from: http://bvtwhue.com.vn/Home/Index/?lang=eng. Accessed November 27, 2020.

22. Dhand N, Khatkar M. Statulator: an online statistical calculator. Sample size calculator for estimating a single mean. 2014. Available from: http://statulator.com/SampleSize/ss1P.htm. Assessed January 2019

23. Sobin LH, Gospodarowicz MK, Wittekind C. TNM Classification of Malignant Tumours. John Wiley \& Sons; 2011.

24. Twisk J, Rijmen F. Longitudinal tobit regression: a new approach to analyze outcome variables with floor or ceiling effects. $J$ Clin Epidemiol. 2009;62(9):953-958. doi:10.1016/j.jclinepi.20 08.10 .003

25. Ministry of health. workshop on health technology assessment in vietnam 2020- develop input database for use in health technology assessment in Vietnam; 2020. Available from: https://moh.gov.vn/tinnoi-bat/-/asset_publisher/3Yst7YhbkA5j/content/hoi-thao-anh-giacong-nghe-y-te-tai-viet-nam-nam-2020-xay-dung-co-so-du-lieu-auvao-su-dung-trong-anh-gia-cong-nghe-y-te-tai-viet-nam. Accessed November 10, 2020, 2020.

26. Tosh JC, Longworth LJ, George E. Utility values in National Institute for Health and Clinical Excellence (NICE) technology appraisals. Value in Health. 2011;14(1):102-109. doi:10.1016/j.jval.2010.10.015

27. Kameyama H, Shimada Y, Yagi R, et al. [Quality of life of patients after colorectal cancer surgery as assessed using eq-5d-51 scores]. Gan to Kagaku Ryoho. 2017;44(12):1083-1085. [Japanese]

28. Huang W, Yang J, Liu Y, et al. Assessing health-related quality of life of patients with colorectal cancer using EQ-5D-5L: a cross-sectional study in Heilongjiang of China. BMJ Open. 2018;8(12):e022711. doi:10.1136/bmjopen-2018-022711

29. Schag CA, Ganz PA, Wing DS, Sim MS, Lee JJ. Quality of life in adult survivors of lung, colon and prostate cancer. Qual Life Res. 1994;3(2):127-141. doi:10.1007/BF00435256

30. Wong CKH, Lam CLK, Poon JTC, Kwong DLW. Clinical correlates of health preference and generic health-related quality of life in patients with colorectal neoplasms. PLoS One. 2013;8(3):e58341. doi: 10.1371 journal.pone. 0058341

31. Färkkilä N, Sintonen H, Saarto T, et al. Health-related quality of life in colorectal cancer. Colorectal Disease. 2013;15(5):e215-e222. doi:10.1111/codi.12143

32. Downing A, Morris EJA, Richards M, et al. Health-related quality of life after colorectal cancer in England: a patient-reported outcomes study of individuals 12 to 36 months after diagnosis. $J$ clin oncol. 2015;33(6):616-624. doi:10.1200/JCO.2014.56.6539

33. Lee Y-H, Kung P-T, Wang Y-H, Kuo W-Y, Kao S-L, Tsai W-C. Effect of length of time from diagnosis to treatment on colorectal cancer survival: a population-based study. PLoS One. 2019;14(1):e0210465e0210465. doi:10.1371/journal.pone.0210465 
34. Neal R, Tharmanathan P, France B, et al. Is increased time to diagnosis and treatment in symptomatic cancer associated with poorer outcomes? Systematic Review. 2015;112(1):S92-S107.

35. Kim SH, Hwang JS, Kim TW, Hong YS, Jo MW. Validity and reliability of the EQ-5D for cancer patients in Korea. Support Care Cancer. 2012;20(12):3155-3160. doi:10.1007/s00520-012-1457-0

36. Li Z, Aninditha T, Griene B, et al. Burden of cancer pain in developing countries: a narrative literature review. ClinicoEconomics Outcomes Res. 2018;10:675-691. doi:10.2147/CEOR.S181192

37. Rauch P, Miny J, Conroy T, Neyton L, Guillemin F. Quality of life among disease-free survivors of rectal cancer. J clin oncol. 2004;22 (2):354-360. doi:10.1200/JCO.2004.03.137

38. Vonk-Klaassen SM, de Vocht HM, den Ouden MEM, Eddes EH, Schuurmans MJ. Ostomy-related problems and their impact on quality of life of colorectal cancer ostomates: a systematic review. Qual Life Res. 2016;25(1):125-133. doi:10.1007/s11136-015-1050-3

39. Bours MJL, van der Linden BWA, Winkels RM, et al. Candidate predictors of health-related quality of life of colorectal cancer survivors: a systematic review. oncologist. 2016;21(4):433-452. doi:10. 1634/theoncologist.2015-0258

40. Chambers SK, Meng X, Youl P, Aitken J, Dunn J, Baade P. A five-year prospective study of quality of life after colorectal cancer Qual Life Res. 2012;21(9):1551-1564. doi:10.1007/s11136-0110067-5

41. Esnaola NF, Cantor SB, Johnson ML, et al. Pain and quality of life after treatment in patients with locally recurrent rectal cancer. $J$ clin oncol. 2002;20(21):4361-4367. doi:10.1200/JCO.2002.02.121
42. Ratjen I, Schafmayer C, Enderle J, et al. Health-related quality of life in long-term survivors of colorectal cancer and its association with all-cause mortality: a German cohort study. BMC Cancer. 2018;18 (1):1156. doi:10.1186/s12885-018-5075-1

43. Briant KJ, Wang L, Holte S, Ramos A, Marchello N, Thompson B. Understanding the impact of colorectal cancer education: a randomized trial of health fairs. BMC Public Health. 2015;15: 1196. doi:10.1186/s12889-015-2499-2

44. Ionescu M, Carey F, Tait I, Steele R. Socioeconomic status and stage at presentation of colorectal cancer. Lancet. 1998;352(9138):1439. doi:10.1016/S0140-6736(98)00052-X

45. Drury A, Payne S, Brady AM. The cost of survival: an exploration of colorectal cancer survivors' experiences of pain. Acta Oncol. 2017;56 (2):205-211. doi:10.1080/0284186X.2016.1266084

46. Dunn J, Ng SK, Breitbart W, et al. Health-related quality of life and life satisfaction in colorectal cancer survivors: trajectories of adjustment. Health Qual Life Outcomes. 2013;11(1):46. doi:10.11 86/1477-7525-11-46

47. Quach DT, Nguyen OT. Clinical, endoscopic and pathological characteristics of early-onset colorectal cancer in Vietnamese. Asian Pacific J Cancer Prevention. 2012;13(5):1767-1770. doi:10.7314/ APJCP.2012.13.5.1767
Patient Preference and Adherence

\section{Publish your work in this journal}

Patient Preference and Adherence is an international, peer-reviewed, open access journal that focusing on the growing importance of patient preference and adherence throughout the therapeutic continuum. Patient satisfaction, acceptability, quality of life, compliance, persistence and their role in developing new therapeutic modalities and compounds to optimize clinical outcomes for existing disease

\section{Dovepress}

states are major areas of interest for the journal. This journal has been accepted for indexing on PubMed Central. The manuscript management system is completely online and includes a very quick and fair peer-review system, which is all easy to use. Visit http:// www.dovepress.com/testimonials.php to read real quotes from published authors. 